Théologiques

Théologiques

\title{
Pratiques de réconciliation au Liban
} Un état des lieux

\section{Pamela Chrabieh}

Volume 23, numéro 2, 2015

Théologies de la réconciliation

URI : https://id.erudit.org/iderudit/1042751ar

DOI : https://doi.org/10.7202/1042751ar

Aller au sommaire du numéro

\section{Éditeur(s)}

Faculté de théologie et de sciences des religions, Université de Montréal

ISSN

1188-7109 (imprimé)

1492-1413 (numérique)

Découvrir la revue

Citer cet article

Chrabieh, P. (2015). Pratiques de réconciliation au Liban : un état des lieux. Théologiques, 23(2), 229-252. https://doi.org/10.7202/1042751ar
Résumé de l'article

Bien que les pratiques de réconciliation connaissent une longue histoire au Liban, celles-ci se diversifient à partir des années 1990. Elles constituent un important objet d'étude pour de nombreux académiciens et académiciennes ainsi que chercheurs et chercheuses ; elles sont devenues la cause commune d'une pléthore d'organismes non-gouvernementaux, d'associations civiles, de mouvements sociaux et d'artistes. Le thème de la réconciliation des Libanais et Libanaises sert aussi de cadre pour le discours politique. Cet article présente un état des lieux de ces pratiques en traitant premièrement de la relation de la réconciliation au dialogue interreligieux puis de la relation de la réconciliation à la mémoire nationale. Il présente par la suite certains exemples au sein de la société civile et identifie en conclusion quelques pistes de réflexion.
Ce document est protégé par la loi sur le droit d'auteur. L'utilisation des services d'Érudit (y compris la reproduction) est assujettie à sa politique d'utilisation que vous pouvez consulter en ligne.

https://apropos.erudit.org/fr/usagers/politique-dutilisation/ 


\title{
Pratiques de réconciliation au Liban
}

\author{
Un état des lieux
}

\author{
Pamela Chrabien* \\ Études du Moyen-Orient \\ Université Américaine de Dubai (Émirats arabes unis)
}

Bien que les pratiques de réconciliation connaissent une longue histoire au Liban, celles-ci se diversifient à partir des années 1990. Elles constituent un important objet d'étude pour de nombreux académiciens et académiciennes ainsi que chercheurs et chercheuses; elles sont devenues la cause commune d'une pléthore d'organismes non-gouvernementaux, d'associations civiles, de mouvements sociaux et d'artistes. En effet, suite à l'arrêt des combats miliciens et militaires en 1989 au Liban et aux accords de Taëf qui ont d'ailleurs été présentés à l'époque comme une tentative de restauration de la paix par un cessez-le-feu et par la réconciliation nationale, le thème de la réconciliation des Libanais et Libanaises sert de cadre pour le discours politique. Cette réconciliation pourrait être qualifiée de mythique puisqu'elle fait fi des différences et de la responsabilité interne: «[N]ous sommes tous des frères [...] nos conflits sont importés »; elle justifie les projets de reconstruction du centre-ville de Beyrouth et constitue la trame de nombreuses actions individuelles et collectives, tant au pays qu'en diaspora.

Cet article n'a pas pour objectif de répertorier toutes les pratiques de réconciliation au Liban, mais il en présente un état des lieux en traitant premièrement de la relation de la réconciliation au dialogue interreligieux

* Pamela Chrabieh est professeure associée en Études sur le Moyen-Orient à l'Université Américaine de Dubai (Émirats arabes unis). Artiste-peintre et activiste pour la paix au Liban et dans la région du Sud-Ouest asiatique, elle a de nombreuses publications sur les arts religieux et la conservation du patrimoine, les dialogues interreligieux et interculturels, la gestion de la diversité, la mémoire de la guerre et la construction de la paix, l'éducation à la paix dans les écoles et les universités, les droits des femmes, etc. Elle a récemment publié: (2016) «Contemporary Feminisms in Lebanon. Lights in the tunnel ", dans M. Raheb, dir., Shifting Identities. Changes in the Social, Political and Religious Structures in the Arab World, Bethlehem, Diyar Publishers, p. 155-172.

C) Revue Théologiques 2015. Tout droit réservé. 
puis de la relation de la réconciliation à la mémoire nationale. Il présente par la suite certains exemples de pratiques de réconciliation au sein de la société civile et identifie en conclusion quelques pistes de réflexion.

\section{Réconciliation et dialogue interreligieux}

Depuis près de trente années notamment, universitaires et chercheurschercheuses s'attèlent à définir le sens et la nature de la réconciliation nationale (al mussalaha al wataniyya). Citons, à titre d'exemples, les travaux du Centre d'études et de recherches sur le Moyen-Orient contemporain (CERMOC) et de l'Institut français au Proche-Orient (IFPO), l'Université Saint-Joseph, l'Université de Balamand, l'Université Libanaise, l'Université américaine de Beyrouth (AUB), les Presses des Paulistes et Al-Makassed. L'ouvrage collectif du CERMOC, intitulé Reconstruction et réconciliation au Liban. Négociation, lieux publics, renouement du lien social (Huybrechts et Douayhi 1999), rassemble les fruits de recherches et de réflexions d'une équipe d'anthropologues, de géographes, de sociologues, de politologues et d'urbanistes; il traite de cas particuliers tels que la reconstruction des villages autour de Saida, dans le sud du Liban, et la mixité sociale sur la Corniche et dans les centres commerciaux de Beyrouth.

D'autres ouvrages publiés en français, en anglais et en arabe ainsi que de nombreuses conférences présentent le même constat et promeuvent le même discours: la réconciliation nationale ne se traduit nullement par le type dit «mythique» car unanimiste/consensuel — même si la poursuite de la lutte politique se fait par d'autres moyens que la guerre physique - mais par la normalisation des relations intercommunautaires et interreligieuses, la reprise de dynamiques métropolitaines et la nécessité de la construction d'une mémoire nationale - en particulier celle de la période 1975-1990 et, plus généralement, la mémoire de la guerre.

En effet, nombreux-nombreuses sont ceux et celles engagés-es dans la voie du dialogue interreligieux qui définissent la réconciliation interreligieuse en tant que "vocation du Liban" et le Liban en tant que "laboratoire interreligieux de la Méditerranée»; d'autres encore, reprenant les propos du pape Jean-Paul II en visite au pays en 1997, soutiennent que la réconciliation interreligieuse constitue le cœur du «Liban message». Cette réconciliation suppose un engagement dans le dialogue interreligieux sur le plan institutionnel, entre le patriarcat maronite et les instances sunnite (Dar al Fatwa) et chiite (le Conseil Supérieur) par exemple, et sur le plan académique, où recherches, publications, déclarations, accords, sommets, 
séminaires de rencontres et colloques se sont multipliés depuis le début des années 1990. Ceux et celles qui prennent part à ces rencontres affirment souvent que le dialogue enrichit les participants et participantes. Il ouvre de nouveaux horizons intellectuels ou apporte une compréhension théologique plus profonde. En ce sens, dialoguer, c'est chercher une voie de partenariat sans renier sa vérité individuelle. L'idée est de constamment chercher à restaurer le lien, et non à le désintégrer. Je cite, à titre d'exemples, les contributions de l'Université Saint-Joseph, des Paulistes, de l'Université de Balamand et des Makassed.

L'engagement dans le dialogue interreligieux se réalise de plusieurs autres façons. J'en signale trois.

1.1 Il y a le dialogue spirituel, incluant l'expérience de la prière interreligieuse, de la méditation et le partage spirituel. Les échanges spirituels renforcent la solidarité entre croyants et croyantes de différentes traditions et les incitent à collaborer aux initiatives des mouvements des religions pour la paix. Je mentionnerai la rencontre, réussie, de prière commune annuelle entre chrétiens et musulmans au collège Notre-Dame de Jamhour, chaque 25 mars en la Fête de l'Annonciation; cette journée est d'ailleurs devenue jour national islamo-chrétien au Liban, symbole de la réconciliation et de la convivialité. Comme j'ai fait partie de la commission chargée de l'organisation de cette rencontre, je suis en mesure de témoigner de son succès; elle dépasse en effet les frontières du Liban et ouvre la voie à une nouvelle culture mariale islamo-chrétienne. Plusieurs actions au sein du pays accompagnent désormais cette rencontre; ainsi eut lieu, en 2013, une manifestation symbolique d'unité à l'initiative de l'organisme non-gouvernemental Offre-Joie: une chaîne humaine relia deux mosquées relevant des communautés sunnite et chiite.

1.2 Il y a le dialogue des œuvres ou de la coopération, c'est-à-dire le dialogue social et éthique qui est l'occasion de se demander comment agir ensemble dans divers dossiers éthiques et comment réfléchir aux questions de société de mondialisation, de génétique, d'écologie, de liberté d'expression, de droits humains, du statut des femmes, etc. Ce dialogue se veut transformateur et n'est pas issu de discussions savantes, autour d'idées et de croyances, ni ne se situe dans la pratique de la prière et de la méditation; il consiste plutôt à travailler avec des personnes d'autres traditions religieuses afin de faire du Liban un lieu où l'on puisse vivre dans la justice et la paix. À certains égards, le travail des théologiens et des experts sert de modèle et d'inspiration à ce genre de dialogue. Des associations islamochrétiennes furent ainsi fondées au Liban dès la première moitié du 
$\mathrm{xx}^{\mathrm{e}}$ siècle; par exemple, la fondation de l'Union pour la protection de l'enfance a vu le jour en 1936 et vise la réhabilitation de jeunes délinquants, toutes confessions confondues. De nos jours, les accords et les actions communes s'établissent contre la guerre, la violence et le terrorisme ou en faveur de la citoyenneté, de la démocratie et des droits humains; pensons au Groupe arabe de dialogue entre chrétiens et musulmans, à Nahwa al-Muwatiniya, à Amam05, à Laique Pride, à Nasawiya, Helem, à Offre-Joie, KAFA, à Women in Front...

1.3 Le dialogue de vie ou le dialogue naturel, c'est-à-dire comment vivre ensemble en convivialité dans les quartiers, les écoles et les universités, dans les milieux de travail, les amitiés, les mariages interreligieux, etc. Il s'agit de plus que la simple coexistence. Cette forme de dialogue n'a jamais revêtu une aussi grande importance. Le dialogue de vie prouve en quelque sorte qu'il ne suffit pas de reconnaitre les fondements et les objectifs du dialogue mais d'adopter une manière d'agir et, plus encore, une manière d'être en développant des relations riches et multiples. La convivialité n'est pas que le fait du hasard, en la commune résidence sur un territoire, mais elle concerne une solidarité qui suppose la possession d'un riche legs de mémoires, de traditions, de coutumes et de croyances ainsi que la volonté de continuer à faire valoir cet héritage tout en y opérant des dynamiques d'échanges et de renouvellement. À cet effet, je cite des extraits de l'ouvrage de la réalisatrice et photographe Nada Raphael, Trait-d'union Islam-Christianisme, ouvrage auquel j'ai pris part en tant que chercheure entre 2007 et 2009 - et qui répertorie des milliers de pratiques du dialogue de vie dans plus de 1200 villages et villes au Liban:

À Haytla-Akkar, certains chrétiens appellent leurs enfants Ahmad suite aux pèlerinages votifs. Le fils d'Elias, Aamer, nous raconte que son ancien collègue de basketball est un chrétien répondant au nom d'Ali. [...] Les chrétiens visitent les maqams (lieu de sépulture d'un prophète ou d'un saint) musulmans des alentours et les musulmans viennent honorer saint Georges.

[...] À Tekrit-Akkar, la mixité prime. Les chrétiens vivent parmi nous (musulmans)... Les mariages interreligieux font partie de notre réalité. Nous avons été éduqués ensemble. Nous avons grandi ensemble, dormi sur les mêmes matelas.

[...] À Al-Mansoura (Ouest de la plaine de la Bekaa), les habitations sont mixtes et il n'y a pas de répartition spatiale des communautés. $\mathrm{Si}$ quelqu'un a besoin d'un don de sang, tout le monde accourt. Le meilleur ami de Mustapha Ali était chrétien et ils s'appelaient "khayyé » (mon frère). Un autre chrétien a demandé à avoir à son chevet son meilleur ami musul- 
man, Bassam, avant de mourir. [...] Souvent à la mort de quelqu'un, les visites de condoléances se font chez les plus proches voisins. Actuellement, les chrétiens bâtissent une salle derrière l'église et les musulmans construisent une autre mosquée. Les chrétiens font des dons à la mosquée et vice versa.

[...] À Kefraya (Koura, Liban Nord), Georgette éduque ses enfants en suivant l'exemple de sa mère qui n'a jamais fait de différence entre musulmans et chrétiens. Mariée en secondes noces à Hassan, elle a de son précédent mariage une fille chrétienne mariée à un musulman qui s'occupe de ses frères comme s'ils étaient les siens et un fils chrétien qui vit à Beyrouth. Avec Hassan, ils ont quatre enfants, tous musulmans [...]. Dans cette famille, les deux religions sont placées sur pied d'égalité et on s'adresse à Dieu avec le cœur avant tout. Au décès de sa grand-mère, mon fils Wassim a récité la Fatiha, le Notre Père et Je vous salue Marie. Les enfants s'entendent tous très bien et lisent autant le Coran que la Bible.

[...] À Bmariam (Caza de Baabda), nous avons toujours vécu ensemble, nous sommes comme des frères. Il y a des mariages mixtes [...]. Durant la guerre de 1980 entre les druzes et les chrétiens, le père de Fadi C., druze [les druzes forment une des communautés religieuses minoritaires au Liban], aide un voisin chrétien, Nicolas, à fuir leur village [...]. Des années plus tard, C. reçoit la visite inopinée de Nicolas avec femme et enfants. (Raphael 2009, $46,67,152,314,411)$

Dans son fameux ouvrage Histoire du pluralisme religieux dans le bassin méditerranéen, Georges Corm (1998) fournit des exemples de réunions communes entre chrétiens et musulmans dans leurs lieux de culte respectifs ainsi que des prises de position communes concernant leur ville ou leur village. Il mentionne aussi des situations relatives au jeûne du Ramadan, durant lequel les populations chrétiennes se gardaient bien d'étaler leurs couverts et se faisaient discrets; il en était de même pour les populations musulmanes lors du carême. Au mois de mai, consacré à Marie chez les chrétiens, il était impensable que la statue de la Vierge, qu'on passait de maison en maison, ne s'arrête pas dans les maisons musulmanes.

C'est assurément en se fondant sur un dialogue de vie que les relations entre les communautés et les individus peuvent continuer à s'épanouir. Le pape Jean-Paul II l'avait bien souligné dans son discours à l'occasion de la remise des lettres de créance de l'ambassadeur du Liban auprès du Saint Siège. Il y invitait les catholiques du Liban de différents rites à être unis entre eux ainsi qu'avec les autres chrétiens et à promouvoir le dialogue avec les musulmans, en particulier le dialogue de la vie, de façon à être des «artisans de paix» (Jean-Paul II 1994). 


\section{Réconciliation et mémoire de la guerre}

Réconciliation et mémoire de la guerre sont intimement liées au Liban, et ce, quelles que soient les appellations données à la guerre: guerre de 19751990 pour certains ou guerre continue pour d'autres, guerre civile, guerre pour les autres, guerre des autres sur le sol libanais, guerre multiforme, etc. ${ }^{1} \mathrm{Or}$, la mémoire nationale de la guerre, dite aussi mémoire officielle ou de l'État, n'a pas encore vu le jour dans ce pays meurtri par des décennies de conflits physiques et de guerres psychologiques ${ }^{2}$. Par contre, les dynamiques paradoxales d'hypomnésie - amnésie ambiante due à l'autoamnistie des «seigneurs de la guerre » — et d'hypermnésie — mémoires collectives communautaristes, confessionnelles, claniques et ethniques prolifèrent. En effet, la loi d'amnistie du 26 août 1991, loi 84, fut élaborée en fonction des critères politiques; elle accorda une amnistie aux criminels de guerre pour tous les actes commis avant le 28 mars 1991, encourageant de ce fait l'établissement d'une omerta, d'une loi du silence. De plus, parce que l'amnistie n'exige aucune manifestation de repentir ou d'amendement de la part de ceux qui en bénéficient, elle n'a qu'un rôle minimal dans l'aménagement des conditions d'une véritable réconciliation.

L'article 3 de la loi 84 stipule que l'amnistie «ne s'applique pas à l'assassinat ou [à] la tentative d'assassinat de personnalités religieuses ou politiques et de diplomates arabes ou étrangers ${ }^{3}$ »; elle est donc conditionnelle. Il s'agit en fait d'une stratégie sécurisante, établie par et pour les responsables politiques et chefs de milices. Le texte de l'amnistie n'accorde à la population libanaise qu'une réconciliation biaisée étant donné que les violations commises dans les années 1970-1980 ne sont pas reconnues et que les responsables politiques ne peuvent être poursuivis. D'ailleurs, la vérité concernant plus de 17000 personnes disparues durant les années 1970-1980 n'est toujours pas connue; or, les disparitions forcées figurent parmi les plus graves crimes, selon la loi internationale. Les familles des personnes disparues n'ont jamais cessé de se battre, jusqu'au dernier souffle même, pour connaître le sort de leurs êtres chers. Toutes leurs

1. Les définitions de la "guerre» au Liban sont nombreuses. J'en présente une analyse détaillée dans deux de mes ouvrages (Chrabieh 2008; 2010).

2. Je renvoie aux travaux du psychiatre libanais Adnan Houballah (2000) et à sa définition de la guerre au Liban, qui inclut les dynamiques physiques et psychologiques alimentant le cycle de violence.

3. Pour plus d'informations à ce sujet, consulter Skaff (2013) ou <https://leportique. revues.org/2686? lang=en>, consulté le 22 juin 2016. 
campagnes se sont heurtées au refus des partis impliqués dans la guerre de rouvrir le dossier ${ }^{4}$.

L'absence de mémoire nationale se traduit aussi par l'absence d'un manuel scolaire commun d'histoire, qui traiterait des événements d'après l'époque de l'indépendance du Liban en 1943. L'unification des programmes d'histoire et d'éducation civique est pourtant indiquée dans le texte des accords de Taëf afin de renforcer le sentiment d'intégration et d'appartenance nationale. À ce jour, les écoles utilisent des manuels scolaires différents et ignorent la période de 1975 à 1990. Chaque communauté possède ses héros et met l'accent sur des événements particuliers. Je me souviens encore des propos du professeur Antoine Messarra - lequel fit partie d'une première commission chargée d'élaborer un programme d'histoire unifié - lorsque j'enseignais à l'Université Saint-Joseph au second cycle dans le cadre d'un programme en relations islamo-chrétiennes; dans une entrevue sur la plateforme en ligne iloubnan.info, celui-ci estime, suite à l'arrêt en 2000 et sous "prétexte politique » du projet d'impression d'un ouvrage commun en histoire, qu'il existe une dichotomie entre l'histoire enseignée et les mémoires collectives: «Le résultat est une société à souvenirs fragmentés, des souvenirs avec lesquels nous ne nous sommes pas réconciliés. » (Messarra 2009)

Pour le juriste Nizar Saghié, le système juridique libanais n'a retenu que la mémoire des détenteurs du pouvoir, dans le but de consolider «le régime charismatique au sens wébérien de l'expression» (Saghié 2005) et de perpétuer les privilèges par héritage, permettant ainsi aux oppresseurs de jouir de l'impunité des crimes qu'ils commettent et de trouver des alibis à leur discours (Galeano 2004). Cette «malmémoire» accorde rarement un rôle à la société civile, à la diaspora libanaise, aux démunis, aux femmes, aux "sans religion", aux non-chrétiens et aux non-musulmans adeptes d'autres religions, aux travailleurs étrangers, aux disparus et à leurs familles; il en va de même pour les réfugiés, pour les jeunes désabusés qui n'arrivent pas à trouver un emploi et qui sont contraints de quitter le pays à la recherche d'un gagne-pain, pour tous ces individus de générations, d'appartenances, de croyances et de pratiques différentes qui ont subi la guerre et en subissent encore les conséquences, tels les fantômes et les cadavres de chiens représentés par Jean-Marc Nahas sur sa fresque Beyrouth mon amour (2005).

4. Pour plus d'informations à ce sujet, consulter les travaux du Centre libanais des droits humains, <http://www.rightsobserver.org>, consulté le 22 juin 2016, ainsi que les rapports d'Amnistie internationale et de Human Rights Watch. 
De 2007 à 2010, j'ai participé à un projet collectif de recherche, par l'Institut Français du Proche-Orient et l'Université Saint-Joseph conjointement, sur la mémoire de la guerre. Les résultats furent publiés dans Mémoires de guerres au Liban (1975-1990) (Mermier et Varin 2010) et les chercheurs et chercheuses y présentent plusieurs constats communs:

1) après la période de 1975-1990, la question de la mémoire de la guerre fut souvent soulevée dans le cadre de colloques ou bien de manière plus diffuse sous la forme d'un devoir éthique et citoyen de "travail de mémoire ", ce qui révèle les enjeux politiques de cette question;

2) la problématique de la mémoire au Liban se réduit à ou se concentre sur la guerre;

3) le contexte libanais est celui

d'un contexte d'inflation mémorielle et de prise en charge par certains acteurs de la société civile de fragments de la mémoire collective à des fins de revendications identitaires, et parfois, victimaires [en plus] d'une fragmentation communautaire et territoriale de la mémoire qui fait écho à l'absence de mémoire nationale ${ }^{5}$;

4) la mémoire n'apparaît pas comme une réserve ou un fonds auquel se nourrit l'histoire mais plutôt comme une manière d'être de la communauté en raison de laquelle cette mémoire est placée au cœur de sa constitution identitaire, sociale et politique;

5) l'enjeu mémoriel est devenu une forme d'action, une stratégie de mobilisation politique portée par de nombreux acteurs aux motivations diverses allant de la simple affirmation d'un positionnement politique dans l'espace public à des revendications spécifiques liées au sort des victimes de la guerre;

6) dans le Liban actuel, un grand nombre de productions académiques et artistiques reflète un rapport difficile au passé, à la guerre en particulier; c'est le cas du théâtre, qui apparaît comme un champ de mise en pratique de la mémoire avec des œuvres fortement engagées et marquées par la guerre: depuis l'œuvre de Ziad Rahbani dans les années 1980 à celles de Rabi' Mroueh et 'Isam Bukhalid suite aux années 1990;

7) la construction d'une mémoire nationale, qui tienne compte de la diversité des mémoires et qui constituerait une plateforme commune, s'avère des plus nécessaires pour l'écriture d'une histoire officielle après l'indépendance du Liban en 1943 - le livre d'histoire officiel s'arrêtant à l'indépendance; elle n'est pas moins importante pour accomplir une véri-

5. Voir <http://www.ifporient.org/en/node/38>, consulté le 22 juin 2016. 
table réconciliation nationale, laquelle constitue une condition essentielle pour de meilleurs liens entre Libanais et Libanaises et pour une meilleure gestion de la diversité sociopolitique.

\section{Pratiques de réconciliation au sein de la société civile}

La majorité des leaders et partis politiques promeuvent un type de réconciliation mythique et unanimiste. Il en existe pourtant un autre qui pourrait être qualifié de "pluraliste» et qui constitue l'objet d'étude de politologues, de sociologues, de journalistes et d'architectes qui ont examiné les impacts de la guerre sur la capitale libanaise et sur la reconstruction de son centre-ville. Je tiens à souligner les travaux de Samir Kassir (2003), d'Ayman Traoui (2003) et de Basilico Gabriele (2003) ainsi que du sociologue Samir Khalaf (2005). Une définition éclairante de ce type de réconciliation est formulée par Joseph Bahout dans Reconstruction et réconciliation au Liban. Négociation, Lieux publics, renouement du lien social:

La réconciliation pluraliste admet le conflit, le régule par le politique et, plus important encore, admet une certaine irréductibilité de l'altérité. Dans la réalité libanaise, cette reconnaissance exprimée du conflit se retrouve sous la forme de la mussalaha tribale ou clanique et met fin au feud - l'affrontement propre à la segmentarité. Le retour de la négociation ou, à tout le moins, du lien social négocié, permet le retour au social et au politique [...]. La réconciliation de type pluraliste, basée sur des conflictualités bien comprises et assumées, ne passe-t-elle pas par une dynamique qui ferait triompher les intérêts sur les valeurs, et qui rejetterait ces dernières hors de la négociation? Tant l'exemple de Bourj-Hammoud [quartier commercial où se retrouvent tous les Libanais et Libanaises mais bastion de la communauté arménienne] que celui de la rue Verdun [quartier commercial de luxe où l'on voit défiler les fashionistas libanaises de toutes confessions mais bastion sunnite], suggèrent une dissolution du marquage communautaire. Il faut cependant rapidement nuancer cette hypothèse, en précisant qu'il ne s'agit pas de dissolution du marqueur identitaire-communautaire dans le marché, comme le posent les thèses — rapides — d'un certain "développementalisme ». Mais au contraire, il y a dissimulation du marqueur identitaire et communautaire à travers les mécanismes du marché, ce qui, dès lors, n'exclut plus une utilisation plus subtile des appartenances respectives dans des stratégies individuelles et collectives de maximisation de pouvoir ${ }^{6}$.

6. Joseph Bahout, «Réconciliation », <http://books.openedition.org/ifpo/4397>, consulté le 22 juin 2016. 
Un exemple de processus de réconciliation pluraliste est celui qui fut engagé au début des années 1990 entre les Forces Libanaises chrétiennes et le Parti socialiste progressiste. Ce processus fut limité au Mont-Liban, au terme de ce qui fut nommé «la guerre de la montagne» (1983-1985) et occasionna le déplacement forcé de plus de 160000 chrétiens. Selon Aida Kanafani-Zahar, dans le cadre de la politique du retour des déplacés menée par le ministère des Déplacés créé en 1992 en conformité avec l'Accord de Taëf, la majorité des chrétiens est revenue dans la région et des réconciliations dites mussalahat entre eux et les druzes ont pu été entamées (Kanafani-Zahar 2005). Toutefois, Kanafani-Zahar critique le recours à ce type de réconciliation comme procédure de résolution des conflits et comme processus institutionnel instauré pour faire revenir les populations déplacées chez elles. En effet, ce processus reposait sur la médiation de chefs politiques et religieux locaux (la pratique coutumière du sulh); il perpétuait, toujours selon Kanafani-Zahar, un ordre social et politique clientéliste qui avait alimenté la guerre, sans donner lieu à l'exercice d'une justice indépendante et au pardon (Kanafani-Zahar 2011).

Un tout autre type de réconciliation, qui se tisse au sein de la société civile et des mouvements populaires, part du bas (bottom up) et articule le plus souvent identités locales et globales (glocal identities). Dans mon livre Voix-es de paix au Liban (Chrabieh 2008), j’ai identifié des pratiques non officielles ou non gouvernementales du ressouvenir en vue de la réconciliation. Ces pratiques, issues de ce que Pierre Nora qualifie de "lieux de mémoire ", ne se réduisent pas à des espaces, des moments, des livres, des édifices et des batailles spécifiques; elles se trouvent aussi dans l'air du temps, dans les courants de pensée, dans les comportements, dans les symboles (etc.) et sont entreprises par divers acteurs et actrices de la société civile et de la diaspora libanaise. De telles pratiques expriment une mémoire de la guerre et se construisent dans l'intimité (intimate spaces of culture, une expression de Herzfeld), encore plus souvent que dans les lieux publics traditionnels. (Herzfeld 2005, 12) Ces pratiques se retrouvent dans l'écriture d'autobiographies et de romans, dans la peinture, dans les bandes dessinées, dans les chansons, dans les hymnes, dans les poèmes, dans la musique, dans le théâtre, dans le cinéma documentaire et de fiction, dans la presse écrite indépendante et dans les mediums électroniques; elles peuvent aussi se trouver sous la forme d'initiatives d'associations locales et transnationales en vue du pardon, de la réconciliation et de la convivialité.

L'État devrait habituellement offrir des espaces de guérison, de socialisation et de transmission de valeurs et de normes de base qui transcendent 
et dépassent les valeurs charriées par la culture de la guerre. Tel n'est pas le cas au Liban. C'est plutôt dans des lieux alternatifs que ces dynamiques se développent, ce que Roel Meijer nomme the street (Meijer 2000,1). Meijer avance l'exemple des mouvements islamistes dans le monde arabe, symbole de la révolution de jeunes, surtout dès les années 1980, en Égypte, en Algérie et au Maroc; il signale aussi des formes de résistance culturelles, telle la musique Rai. Les jeunes interviewés dans le cadre de la recherche dont fait état mon livre Voix-es de paix au Liban développent des lieux alternatifs ou tentent de prendre en charge leurs souffrances et de nommer l'innommable. Par contre, d'autres Libanais et Libanaises ne font pas de même, animés plutôt par le désir de se décharger le plus rapidement possible de leurs plaies; cela ne permet pas de faire le travail de mémoire et de réconciliation nécessaires à la reconstruction nationale. En d'autres termes, plus la souffrance est refoulée, plus la chance d'une véritable réconciliation au plan national est enterrée. Plus les traumatismes ne sont pas traités, plus «les chances que la boîte de Pandore des haines alimentées par la guerre s'ouvre augmenteront" - citation d'un interviewé - et plus l'édifice de la convivialité se lézarde, se fissure et risque de s'effondrer. Plus on évite d'aborder le sujet de la guerre en profondeur dans tous les secteurs de la société, y compris le secteur académique (scolaire et universitaire), plus tout ce qui sera transmis aux nouvelles générations se résume à l'incompréhension et à l'ignorance de l'autre.

Un premier exemple de pratiques de réconciliation réussies que je tiens à mentionner est celui des travaux de la psychothérapeute Alexandra Asseily, fondatrice du Jardin du Pardon au centre-ville de Beyrouth; je souligne notamment son initiative, en novembre 2005, en partenariat avec la Young President's Organization: "A Journey of discovery, understanding and healing». L'objectif de ce projet était de créer des lieux de dialogue interspirituel et interculturel entre une délégation d'Américains ayant perdu leurs proches le 11 septembre 2001 et des Libanais et Libanaises. Divers thèmes furent abordés dans le cadre de cercles d'échanges: conflits religieux, mémoires meurtries et processus de convalescence, histoires de vie en lien avec le terrorisme ou la guerre, destruction et reconstruction (physique et psychologique), deuil, pardon et paix, etc. Ce projet eut lieu dans trois villes au carrefour d'identités plurielles et dont l'histoire est mouvementée: Beyrouth, Byblos-Jbeil et Sidon-Saida ${ }^{7}$. Madame Asseily a

7. Je note aussi la contribution de plusieurs groupes comme Offre Joie qui a organisé un sit-in en faveur de la paix en février 2007 au Musée national de Mathaf au Liban 
organisé également en novembre 2011 une conférence internationale au Liban sur la guérison des blessures du passé à laquelle j’ai participé: «Healing the Wounds of History: Addressing the Roots of Violence », en partenariat avec le Centre for Lebanese Studies (Oxford), la GuerrandHermès Foundation for Peace et l'Institute of Diplomacy and Conflict Transformation (Lebanese American University) ${ }^{8}$; elle a aussi pensé à une cérémonie interreligieuse au Jardin du Pardon, au centre-ville de Beyrouth; la rencontre «Ensemble autour de Marie » a été réalisée en mars 2013. Les participants et les participantes à la conférence et à la cérémonie ont abordé plusieurs questions dont les suivantes: comment gérer le douloureux héritage de la violence passée - et présente - tout en préservant la fragile harmonie sociale ou tout en développant de nouveaux liens conviviaux? Doit-on et est-il possible de traduire en justice les auteurs des violations et des massacres et ainsi combattre la culture d'impunité ou est-il plus important et pensable - mesures pragmatiques — de s'atteler d'abord à des mesures réalistes qui favorisent la paix? Dans cette perspective, quel est le rôle que la société civile devrait jouer et quelles sont les responsabilités des acteurs et des actrices non étatiques de la paix?

À l'issue de ces activités alliant discours académiques sur la mémoire de la guerre et la réconciliation et pratiques de réconciliation, les participants et les participantes étaient d'accord sur l'importance de promouvoir les mécanismes sociaux traditionnels, telle la pratique de la mussalaha (réconciliation) dans les villages mixtes, et de renforcer l'éducation nonofficielle à la paix, en utilisant les ressources humaines sous-estimées de gestion de conflits et qui se trouvent déjà au sein de la société civile et de la diaspora libanaises. Plusieurs jeunes activistes qui prirent part à ces activités ont poursuivi leur engagement individuel pour la paix, soit en fondant de nouveaux organismes non-gouvernementaux, soit en rejoignant des organismes internationaux, soit en utilisant les plateformes virtuelles comme champ de lutte parallèle aux manifestations dans les rues. De plus, Healing the Wounds of History est devenu une association organisant continuellement, dans plusieurs villes au Liban, des ateliers de réconciliation et de guérison des blessures; l'association utilise des techniques éduquant à l'auto-guérison et l'interaction sociale, tels l'écoute mutuelle, les dialogues intérieurs et entre personnes ou groupes, l'art thérapie, les exer-

ainsi que des activités continues favorisant le dialogue interreligieux, telles des camps de jeunes et la restauration de façades d'immeubles dans des quartiers défavorisés et des quartiers portant les stigmates physiques de combats et d'attentats à la voiture piégée.

8. Voir <http://www.healingwoundsofhistory.org>, consulté le 22 juin 2016. 
cices d'énergie physique (bodily energy exercises), la connaissance «incarnée " (embodied knowledge), l'intervention thérapeutique par la nature, etc.

Un deuxième exemple de pratiques de réconciliation réussies est celui des excuses publiques émises par d'anciens miliciens chrétien et musulman, Assaad Chaftari et Muhieddine Mustapha Chehab, que j'ai eu la chance et l'honneur de rencontrer et d'écouter à Montréal en 2005, et plus tard au Liban. Depuis des années, Chaftari et Chehab parcourent le Liban et plusieurs pays à travers le monde afin de témoigner de leur mémoire de guerre, de leur réconciliation et de leur cheminement vers la paix et la convivialité (Initiatives of change 2002). Leurs perceptions de la différence religieuse furent transformées par leurs engagements au sein de l'organisation internationale du Réarmement Moral — qui devint par la suite Initiatives et changement. Les paroles de Chaftari lors d'une conférence prononcée sur la paix au Musée de la Soie à Bsouss au Liban en novembre 2005 sont mémorables: "Je ne peux rendre la vie à ceux que j’ai tués, mais je peux demander et œuvrer pour le pardon" ${ }^{9}$. Chehab a fondé en 1992 un comité social qui promeut le dialogue; ses travaux montrent l'importance de ne pas oublier les déboires de la guerre afin de briser la «chaîne de la haine ${ }^{10}$ ». Chaftari a récemment publié un livre intitulé La vérité même si ma voix tremble (Chaftari 2015), dans lequel il raconte sans fard son histoire et répond à un devoir de mémoire.

Le parcours de Chaftari est retracé dans le documentaire Sleepless nights d'Eliane Raheb (2012). Sincère dans ses excuses, Chaftari y affirme vouloir aider les familles des disparus de la guerre. Parmi elles, Maryam Saiidi, sans nouvelles de son fils depuis 1982. Il avait 16 ans et combattait avec le parti communiste. Elle n'a pas encore trouvé la paix intérieure et cherche toujours à connaître la vérité. C'est vers Chaftari qu'elle se tourne pour obtenir des réponses. Eliane Raheb, la réalisatrice, explique:

Au début, je me suis concentrée sur Assaad Chaftari. Je voulais savoir qui était derrière ce personnage. Je voulais savoir comment on peut ressortir du processus de la guerre une fois qu'on y est entré, comment on retrouve son humanité et on redevient civil. Finalement, Maryam est entrée dans le documentaire et [est] devenue un deuxième personnage, comme un élément vivant du passé et du présent d'Assaad ${ }^{11}$.

9. Propos cité de mémoire, notes personnelles.

10. Pour plus de détails sur les travaux de Chaftari et de Chehab, consulter l'excellent mémoire de maîtrise de Haugbolle (2002).

11. «Sleepless Nights: l'amnésie de la guerre civile libanaise», Euromed Audiovisuel, 22 avril 2013, <http://euromedaudiovisuel.net/p.aspx ? t=news\&mid=21\&cid=15\& l=fr\&did=1384>, consulté le 22 juin 2016. 
Le documentaire ne montre aucun des personnages comme étant totalement coupable ou totalement victime. Le champ des complexités liées à la guerre, aux traumatismes et à l'intimité de la souffrance y est bien exploré. La réalisatrice affirme n'avoir pu percer ce qu'elle appelle le «mystère Assaad ", mais elle reconnaît l'unicité de son message et de sa pratique puisque rares sont les ex-miliciens ayant fait leur mea culpa au Liban. Dans son blog "Chroniques civiles du Liban et d'ailleurs", la blogueuse et activiste Souha Tarraf mentionne l'importance du message que porte Chaftari:

Il est urgent de ne pas oublier, urgent de regarder en arrière et d'essayer de (nous) comprendre enfin entre nous, les citoyens de ce pays. Urgent de saluer le courage de gens comme Régina Sneifer et Assaad Chaftari qui portent leur passé d'acteurs de la guerre comme une croix et passent le seul message qui vaille: celui de la réconciliation civile, citoyenne ${ }^{12}$.

Outre Eliane Raheb, plusieurs cinéastes et réalisateurs - Lamia Joreige ( Objects of War», 2000-2003-2006), Jean Khalil Chamoun ( «In the Shadows of the City», 2000), Danièle Arbid («Dans les champs de bataille», 2004), Josef Fares ( "Zozo», 2005), Khalil Joreige et Joana Hadjithomas ( $A$ A perfect day», 2005), Ziad Doueiri ( "West Beirut», 1998), Nada Raphaël ( "Juillet 2006. Un mois inoubliable dans l'histoire du Liban. Flash...Back? Ou Ba3dein?») — montrent que la mémoire de la guerre comporte des histoires personnelles exprimant le vécu intérieur de l'horreur et reflétant ce que nous pouvons qualifier de « devoir des survivants ». Ces jeunes cinéastes insistent sur le récit de chacun de leurs personnages, leur relation avec le passé, leur façon de voir et de gérer le présent. À cet égard le long-métrage «A perfect Day» est particulièrement significatif parce qu'il soulève des questions pour le moins importantes:

Que faire de la mémoire? Que faire des disparus? Comment gérer le présent quand notre passé est aussi complexe que singulier? Dans la vie comme dans le film, chacun lutte à sa manière. Cette observation d'une nation déchirée entre le souci de préserver la mémoire et la tendance à la rejeter est brillamment incarnée par les acteurs du film. (Demirdjian 2006)

Les pièces de théâtre traitant de la guerre, de la mémoire et de la réconciliation sont aussi nombreuses que les films et les documentaires. Celle du dramaturge libano-québécois Wajdi Mouawad, intitulée «Littoral », traite de secrets familiaux, de charniers, de fosses communes et de squelettes

12. «Une paix si malaisée! Comme une litanie...», 19 novembre 2013, <https:// libanchroniquesciviles.wordpress.com/tag/assaad-chaftari>, consulté le 22 juin 2016. 
dans les placards. La pièce de théâtre "Amour et guerre sur le toit: une histoire tripolitaine" a été produite en 2015 par l'organisation libanaise non gouvernementale contre la censure $\mathrm{MARCH}^{13}$; elle rassemble 16 jeunes Tripolitains des quartiers antagonistes de Bab el-Tabbané et de Jabal Mohsen, qui ont été initiés au dialogue, à la tolérance et à la réconciliation par le biais de l'expression théâtrale. Le rôle des arts dans l'expression des représentations et des sentiments concernant les situations passées et actuelles de la société libanaise est crucial. Dans cette perspective, une multitude d'artistes libanais sont engagés à promouvoir une culture de la paix, de la réconciliation et de la convivialité en recourant à des styles variés d'expression - du minimalisme à l'onirisme ${ }^{14}$. La scène artistique libanaise constitue d'ailleurs un creuset d'innovations en matière de construction de mémoires individuelles et collectives de la guerre, et ce, depuis le milieu des années 1990.

Un troisième exemple de pratique de réconciliation réussie est celui de travaux de plus d'une centaine de blogueurs et de blogueuses, depuis 20052006 notamment. Lors de l'été 2006, plusieurs ont érigé des chroniques de vie et de mort pour dénoncer trente-trois jours de bombardements, de violences et d'agressions physiques et morales. Selon le caricaturiste et blogueur Mazen Kerbaj (2007),

[d]ès que le conflit a éclaté, je me suis mis à dessiner. C'était ma manière à moi de résister. Bien sûr, je n'étais pas assez naïf pour croire que l'art pouvait vaincre la guerre mais, par contre, il pouvait lui survivre. Il ne fallait surtout pas me laisser vampiriser par l'ignominie de la situation. Se tenir en marge de l'événement tout en devenant témoin, que ce soit par l'écriture ou le dessin, tel était mon objectif premier.

L'apparition du self-media lors des combats de l'été 2006 a traduit un phénomène de multiplication des acteurs et des actrices qui jouent un rôle considérable en matière de perception et d'influence. Certains acteurs ont contribué à ce que de nombreux commentateurs et journalistes nommèrent «la première bloguerre mondiale »; cette guerre virtuelle préfigure de nouvelles formes d'affrontements et constitue à l'évidence un nouveau théâtre

13. Voir <https://www.marchlebanon.org>, consulté le 22 juin 2016.

14. À titre d'exemples: Ziad Abillama, Fadia Kisrwani Abboud, Maissa Alameddine, Oussama Baalbaki, Rita Baddoura, Gregory Buchakjian, Rowina Bou Harb, Hala Dabaji, Maher Diab, Ghassan Ghazal, Lina Hakim, Joanne Issa, Maria Kassab, Zena el-Khalil, Joumana Mattar Moukarzel, Lena Merhej, Chantal Partamian, Amanda Riffo, Marie-Joe Raidy, Fouad Shehab, Elise Tabet, Rayanne Tabet, Jana Traboulsi, Wafa Wafa et Hassan Yateem. 
d'opérations visant à créer, à façonner et à modifier les diverses représentations d'un conflit. Il suffit de penser à Loubnan Ya Loubnan, My Lebanon, Peace4Lebanon et Lebanon Under Attack. D'autre part, certains de ces acteurs ont créé une culture de convivialité plaidant pour la paix. On peut penser à Bloggingbeirut, Letters Apart, Pamela Chrabieh Badine, Chroniques Beyrouthines et Pour que le Liban vive ${ }^{15}$.

Dans un article que le journal libanais francophone L'Orient-le-Jour a publié le 15 décembre 2015, je rendais notamment hommage aux blogueurs et aux blogueuses de 2006, lesquels continuent d'influencer l'opinion publique libanaise en utilisant le blog mais aussi diverses autres plateformes telles que Facebook, Twitter, Instagram et YouTube:

Après avoir cru à l'existence d'une période qualifiée de post-guerre, mes collègues et moi-même nous étions rendu compte dès 2005 de l'ampleur des divisions internes lesquelles perduraient, de la guerre psychologique qui alimentait continuellement la guerre physique [...]. Cette fois, il fallait absolument témoigner de ce qui se passait avec sons, images et paroles, élever la voix et la porter plus loin, pour ne pas oublier et pour ne pas devenir les oubliés de l'histoire. Avec l'été 2006, la mise en mémoire devint une entreprise démocratisée (democratized), diversifiée, exponentielle, voire virale (viral). Les espaces publics comme lieux de médiation entre les pouvoirs étatiques et les citoyens s'élargirent, favorisant une participation beaucoup plus active qu'auparavant, des dynamiques alternatives moins hiérarchisées, une meilleure visibilité aux diverses expressions d'individus et de collectivités, et la construction de liens transnationaux. Avec le blog, les contraintes des médias traditionnels de la censure étaient contournées et de nouveaux modes de sociabilité se mettaient en place pour contribuer à redéfinir la configuration du paysage sociopolitique, religieux et économique ${ }^{16}$.

Dix ans plus tard, plusieurs blogueurs et blogueuses publient des articles sur des sujets divers, de la mode à la cuisine et en passant par la politique régionale, les arts et les carnets de voyage. Toutefois, certains sont des agents de paix et contribuent, grâce à leurs écrits et aux débats qui s'en suivent, à l'éducation non officielle à la paix; ils suscitent la dis-

15. Pour plus d'informations, je suggère l'excellente étude, publiée en février 2007, «Une illustration de la guerre de l'information: le conflit entre Israël et le Hezbollah de l'été 2006 », <www.ege.fr/download/liban2007.pdf>, consulté le 22 juin 2016. L'étude analyse l'irruption des blogosphères libanaises et israéliennes lors de l'été 2006, ce qui illustre l'importance de l'échiquier informationnel dans le cadre d'un conflit armé et des innovations en modifiant la structure.

16. Chrabieh, "Pourquoi bloguer?", <http://www.lorientlejour.com/article/960252/ pourquoi-bloguer-.html>, consulté le 22 juin $2016 s$. 
cussion sur les droits des femmes, sur la liberté d'expression, sur la corruption étatique, sur le dialogue interreligieux, sur le confessionnalisme et la laïcité, sur la démocratie, sur la justice transitionnelle, etc. Certains font partie de mouvements ou d'organisations luttant pour des causes communes intercommunautaires. Ainsi en est-il de Vous puez! (You Stink), qui dénonce le laxisme gouvernemental dans la crise des déchets au centre-ville de Beyrouth depuis juillet 2015, de MARCH pour la liberté d'expression et la réconciliation de jeunes Alawi et Sunnites à Tripoli au Nord du Liban, de Beirut Madinati lors des élections municipales de mai 2016 et de Act for the Disappeared, qui répertorie les milliers de disparus forcés des années 1970-1980-1990 et livrés aux mains des milices libanaises et des armées syrienne et israélienne.

\section{Pistes de réflexion}

Les constructions non officielles de la mémoire - individuelle et collective - de la guerre montrent que les Libanais et Libanaises ayant vécu et survécu à la guerre tentent d'en relater les souvenirs transmis d'une génération à une autre, et ce, non sans grandes difficultés compte tenu des logiques de l'impensé et de l'impensable de la guerre. De leurs œuvres littéraires, artistiques et médiatiques ainsi que de leurs actions au sein de groupes et d'organisations de la société civile et de la diaspora irradie une énergie de paix. Il est évident que les pratiques signalées entre autres par mon propos ne constituent pas le remède miracle ni ne peuvent remplacer le système judiciaire, le rôle de l'État et celui de l'éducation officielle; elles les complètent toutefois, en offrant un véritable potentiel de promotion de la réconciliation et de la culture de la paix. Un des exemples les plus pertinents à cet égard est celui des réseaux sociaux, le Liban possédant le plus haut taux de pénétration d'Internet et de Facebook en Asie du Sud-Ouest — plus de $80 \%$ et $67 \%$ de la population, respectivement (Internet World Stats 2015). Les réseaux sociaux constituent des espaces privilégiés pour la conscientisation et la dissémination en masse de savoirs et de pratiques. Un des récents mouvements populaires en ligne, (Vous puez) rassembla en 2015-2016 des milliers de Libanais et Libanaises — qui manifestèrent à plusieurs reprises, au centre-ville de Beyrouth, pour réclamer une solution écologique à la crise des déchets.

Certaines pratiques de réconciliation de la société civile libanaise sont basées sur des modalités locales et traditionnelles de gestion de conflits, de médiation et de pardon; d'autres suivent le développement mondial d'une 
culture des droits humains, laquelle a fait éclore un nouveau combat qu'est celui contre l'impunité. Le mouvement "Nous voulons responsabiliser" (Badna Nhasseb) et le Comité des familles de disparus constituent actuellement des exemples de lutte non officielle contre la culture de l'impunité qui sévit au Liban. Un autre exemple est celui du Centre libanais des droits humains, qui reprend et poursuit sur le terrain les activités de l'association SOLIDA (Soutien aux Libanais détenus arbitrairement) fondée en France en 1996. Ce centre traite des dossiers épineux de la torture, des détentions arbitraires, des disparitions forcées et de l'impunité des violations graves des droits de l'Homme. Il fournit une prise en charge légale aux victimes et à leurs familles et effectue un travail d'enquête et recueille des témoignages tout en diffusant des informations par le biais de communiqués de presse et de rapports. L'association a ouvert, en octobre 2007, un autre centre d'accueil et d'assistance pour les victimes de la torture et leurs familles - le Centre Nassim — qui propose une prise en charge pluridisciplinaire - médicale, psychologique, sociale, professionnelle et juridique $^{17}$. À la fin des années 2000, le mouvement «Notre unité est notre salut» (Wahdatouna Khalasouna) établit des liens avec des Commissions Vérité et réconciliation, dont celle que présidait l'archevêque Desmond Tutu en Afrique du Sud.

Des organismes, tels le Centre libanais des droits humains, subissent souvent de l'intimidation de la part des forces de sécurité interne et se heurtent à de nombreux obstacles, comme le non-accès aux archives de l'État et des ex-milices. Les membres de ce centre subissent régulièrement diverses formes de harcèlement, même d'ordre judiciaire. En fait, le débat public sur la réconciliation oppose la majeure partie des responsables politiques à des membres de la société civile. De plus, les contingences politiques empêchent l'accomplissement du devoir de justice ou limitent celui-ci à des cas particuliers, tel celui du Tribunal spécial pour le Liban (TSL) qui a tenu le procès in absentia des auteurs présumés de l'assassinat de l'ancien premier ministre Rafic Hariri en $2005^{18}$. En effet, ce tribunal ne constitue pas le fruit d'une entente politique interne. Certains partis politiques libanais imputent cet assassinat à la Syrie tandis que d'autres nient farouchement l'accusation. Sous les mandats de Jacques Chirac et de

17. Le Centre libanais des droits humains, <http://www.rightsobserver.org>, consulté le 22 juin 2016.

18. Voir le guide pratique «Les processus de transition, justice, vérité et réconciliation dans l'espace francophone», publié par la Direction de la paix, de la démocratie et des droits de l'Homme (2013). 
Georges W. Bush, la France et les États-Unis avaient cherché à obtenir, par une résolution du Conseil de sécurité des Nations Unies, la création en juin 2007 du TSL. La mission de ce tribunal n'est nullement de contribuer à la réconciliation nationale mais de juger les responsables de cet attentat et d'autres survenus après le $1^{\text {er }}$ octobre 2004 . De plus, l'institution du TSL

est perçue par ses détracteurs comme un facteur d'aggravation de la crise interne et l'instrument d'une politique jugée colonialiste de la part de puissances occidentales et de l'ONU. De vives critiques se font entendre à l'encontre de l'unilatéralisme de son institution [...] et de l'immixtion dans les affaires internes libanaises qu'elle implique. (Lelarge 2007)

Par ailleurs, le débat public oppose des membres de la société civile entre eux. Une des questions qui divisent actuellement le plus porte sans conteste sur les moyens d'équilibrer les demandes de justice et de vérité et celles politiques. Une autre question concerne le type de justice à promouvoir: justice punitive ou bien justice transitionnelle en l'occurrence. Certains organismes, tel le Centre libanais des droits humains, croient aux effets positifs des poursuites judiciaires et soutiennent l'argument de l'obligation morale de juger et de punir les coupables, même si ceux-ci font partie de l'appareil étatique, car c'est précisément une réparation qu'attendent les victimes. De plus, les poursuites judiciaires permettent d'éradiquer la perception erronée selon laquelle une ou des communautés entières - communauté maronite, sunnite, chiite, druze ou autres — sont responsables de la violence et des atrocités. Pareilles poursuites sont également considérées comme un moyen de persuasion efficace contre les futures violations et une garantie plus sûre contre l'installation et la perpétuation de la violence. D'autres organismes comme Offre-Joie, Ensemble autour de Marie et Healing Wounds of History n'incluent pas les poursuites judiciaires dans leurs missions.

J'ajoute que la plupart des organismes non-gouvernementaux au Liban opèrent en mode "tour d'ivoire». Face à ce morcellement, il est évident que le débat en cours ne porte pas nécessairement plus de fruits que ceux récoltés par des pratiques ponctuelles. Chaque pratique pourrait mettre à contribution pour elle-même les idées des collectivités les plus au fait d'une problématique particulière, bien mieux qu'une perspective unique pour chapeauter tout. Toutefois, les efforts séparés diluent cette force engendrée par la société civile et la diaspora; ils engendrent une concurrence des loyautés, des priorités, des ressources et du prestige. À cet effet, il est nécessaire de constituer ce que Michael Albert (2004, 89) appelle un «Mouvement solidarité dans l'autonomie» (MSA), qui consiste à cumuler 
les objectifs de groupes et d'individus affiliés tout en assurant le respect de leurs différences et leur autonomie. Parce que travailler ensemble implique généralement de fonder une coalition, on cherche alors les points communs entre les programmes et les analyses de tous ces alliés potentiels. La dynamique visée est celle d'une fusion des différents objectifs des parties au sein d'un cadre élargi durable, lequel rend possible des efforts nationaux et permet un soutien mutuel tout en gardant intacts les objectifs spécifiques des parties pour la poursuite des pratiques individuelles et collectives.

Par ailleurs, plusieurs experts et activistes pointent du doigt une mentalité confessionnaliste (communautariste, cloisonnant tout) et le système sociopolitique de même acabit. Ils proposent de réformer le système, de « dés-instrumentaliser» le religieux par le politique et vice-versa, d'œuvrer à changer les mentalités ou encore d'abolir les confessionnalismes tant politique qu'individuel. Le Centre international pour la justice transitionnelle affirme qu'une réelle paix et une véritable réconciliation nécessitent à la fois une réforme institutionnelle visant à éliminer le partage du pouvoir sur une base confessionnelle et la responsabilisation des puissances étrangères, lesquelles - Israël et la Syrie notamment — ont joué un rôle majeur dans la guerre.

Il y a certes des aspects positifs de cette gestion de la diversité: la reconnaissance d'une diversité religieuse et confessionnelle de même que la préservation des droits et privilèges de communautés minoritaires. En dépit de ces aspects positifs, la gestion de cette diversité est en crise depuis plusieurs décennies. Elle souffre en effet de problèmes internes au système et de bien mauvaises applications: mauvaise gouvernance, clientélisme confessionnel vis-à-vis de puissances régionales et internationales, corruption de l'administration de l'État, promotion du religieux comme facteur identitaire, ancrage sociopolitique exclusifs... Le système sociopolitique confessionnaliste est censé promouvoir la démocratie consociative, mais il n'assure pas la reconnaissance de l'égalité entre Libanais et Libanaises. Cette reconnaissance est restreinte aux communautés auxquelles ces derniers doivent impérativement appartenir. Georges Corm (1986) a bien souligné, dans une œuvre majeure sur le Liban, que la culture de discorde est intrinsèque au système sociopolitique et a pour but d'empêcher toute forme de coopération et de respect entre les communautés, mais aussi tout espoir de paix pourtant si déterminant pour le développement. Selon Ziad Hafez,

Le confessionnalisme, forme aiguë du factionnalisme, est donc, à plus d'un titre, antinomique avec la démocratie. Il est même totalitaire dans son essence, empêche le développement d'une communauté de citoyens et étouffe 
toute velléité d'indépendance. De plus, il s'appuie sur une culture de la peur et du soupçon. Pire encore, il est foncièrement raciste car il promeut une image péjorative de l'Autre. Il est fondé sur l'illusion fallacieuse que la communauté est l'ultime protectrice de ses membres, ce qui est une contradiction en soi du fait que les droits individuels sont subordonnés à ceux de la communauté. De plus, l'histoire a démontré d'une manière irréfutable que le confessionnalisme a engendré, et continuera de le faire, la violence parmi les citoyens. Enfin, la subordination des droits individuels à ceux de la communauté affaiblit les libertés individuelles et limite la responsabilisation et la demande des comptes. Ces libertés individuelles sont prises en otage par les chefs confessionnels [...]. La culture confessionnelle ne permet pas l'établissement d'un État fort, c'est-à-dire un État où les institutions sont établies à la suite d'un contrat social légitimant l'État et ne reconnaissant d'autre autorité que celle des institutions de l'État. L'objet de l'interaction politique au Liban est tout simplement le partage du pouvoir et non l'établissement de l'État. Ainsi, les fonctions administratives et politiques sont distribuées en fonction des intérêts des seigneurs de la guerre et de leurs alliés, les financiers. Quant au pouvoir décisionnel, il se trouve bien souvent entravé, rendant par exemple difficile la mise en œuvre des politiques publiques porteuses de développement économique et social. (Hafez 1986)

Compte tenu des obstacles identifiés ici et bien d'autres encore, l'avenir de la réconciliation nationale apparaît, de prime abord, obscur. "Lorsque les grandes nations brillent par leur devoir de mémoire, notre pays scintille par son amnésie », clamait Saër Karam en 2005. À ce jour, une histoire nationale fragmentaire est transmise, perpétuant les tabous, concoctant des identités meurtrières qui trahissent et pulvérisent le fameux adage «Plus jamais ». Selon Eduardo Galeano, «l'impunité du pouvoir, fille de la malmémoire, est une des maîtresses de l'école du crime ${ }^{19}$ ». Cette école est malheureusement fréquentée par un nombre croissant de jeunes Libanais et Libanaises. De plus, il semble peu probable que les dirigeants politiques actuels, dont certains sont présumés coupables d'atrocités, vont établir une commission Vérité et réconciliation pour examiner leurs actes. Toutefois, les pratiques réussies de membres de la société civile et le dynamisme de cette dernière contribuent, quoiqu'à petits pas, à transformer les rapports sociaux et politiques; elles constituent le socle sur lequel peut être construit un autre avenir, plus convivial et libérateur.

19. Eduardo Galeano, «Mémoires et Malmémoires », <http://lionel.mesnard.free.fr/le \% 20site/Eduardo-Galeano.html>, consulté le 22 juin 2016. 


\section{Références}

Albert, M. (2004), L'élan du changement. Stratégies nouvelles pour transformer la société, Montréal, Écosociété.

Chaftari, A. (2015), La vérité même si ma voix tremble. Récit d'une rédemption et d'une renaissance, Beyrouth, Éditions Dergham.

Chrabieh, P. (2008), Voix-es de paix au Liban. Contribution de jeunes de 25-40 ans à la reconstruction nationale (Interaction islamo-chrétienne), Beyrouth, Dar el-Machreq, Institut d'études islamo-chrétiennes de l'Université Saint-Joseph de Beyrouth.

(2010), Quelle gestion des diversités au Liban? Du confessionnalisme au pluralisme médiateur, Sarrebruck, Éditions universitaires européennes.

Corm, G. (1986), Géopolitique du Conflit Libanais, Paris, Éditions La Découverte.

(1998), Histoire du pluralisme religieux dans le bassin méditerranéen, Paris, Société nouvelle Librairie orientaliste Paul Geuthner.

Demirdjian, D. (2006), " “A Perfect Day”, une réflexion envoûtante sur la mémoire, le passé et le présent", L’Orient-le-Jour, Beyrouth, 22 avril (archives électroniques).

Direction de LA PAIX, DE LA DÉmocratie ET DES DROITS DE L'HOMME (2013), Paris, <http://www.francophonie.org/IMG/pdf/guide-oif-tjvrbatweb1003.pdf>, consulté le 22 juin 2016.

Gabriele, B. (2003), Beyrouth 1991-2003, Paris, Le point du Jour.

Galeano, E. (2004), Mémoire de feu, Paris, Plon.

Hafez, Z. (2009), «La rente et le confessionnalisme au Liban », Confluences Méditerranée, 70, p. 89-103, <https://www.cairn.info/revue-confluencesmediterranee-2009-3-page-89.htm>, consulté le 22 juin 2016.

Haugbolle, S. (2002), Collective Memory of the Civil War in Lebanoni, St. Antony's College, University of Oxford, <http://www.111101.net/ Writings/Author/Sune_Haugbolle>, consulté le 22 juin 2016.

Herzfeld, M. (2005 [1997]), Cultural Intimacy. Social Poetics in the Nation-State, éd. révisée, New York, Routledge.

Houballah, A. (2000), Le Virus de la violence, Paris, Albin Michel.

Huybrechts, E. et C. Douayhi (1999), Reconstruction et réconciliation au Liban. Négociation, lieux publics, renouement du lien social, Beyrouth, Publications de l'Institut Français du Proche-Orient. 
Initiatives of Change (2002), Breaking the Chain of Hate. Agenda for Reconciliation, London, [s.é.].

INTERNET World Stats (2015), <http://www.internetworldstats.com/ middle.htm\#lb>, consulté le 22 juin 2016.

Jean-Paul II (1994), Discours du Saint-Père Jean-Paul II au nouvel ambassadeur du Liban près le Saint-Siège, S. Exc. M. Youssef Arsanios, à l'occasion de la présentation des lettres de créances, <https://w2.vatican. va/content/john-paul-ii/fr/speeches/1994/october/documents/hf_jp-ii_ spe_19941001_ambasciatore-libano.html>, consulté le 22 juin 2016.

Kanafani-Zahar, A. (2005), "Réflexion sur le blocage des "dernières réconciliations" au Mont Liban ", Cahiers de la Méditerranée, 71, p. 129-143.

Kanafani-Zahar, A. (2011), Liban, la guerre et la mémoire, Rennes, Presses universitaires de Rennes.

Karam, S. (2005), "En aparté ", La Revue du Liban, 10 octobre 6 novembre, p. 12, <http://www.rdl.com.lb/2010/q4/4286/En_Aparte. pdf>, consulté le 22 juin 2016.

Kassir, S. (2003), Histoire de Beyrouth, Paris, Fayard.

Kerbaj, M. (2007), L'Orient-le-Jour, Beyrouth, 9 mars (archives électroniques).

Khalaf, S. (2005), Heart of Beirut. Reclaiming the Burj, London, Saqi Books.

Lelarge, A. (2007), «Le tribunal spécial pour le Liban », Annuaire français de droit international, 53/1, p. 397-428.

Mermier, F. et C. Varin (2010), dir., Mémoires de guerres au Liban (19751990), Paris, Sindbad-Actes Sud.

Messarra, A. (2009), «Écoles au Liban. Dur d'enseigner une histoire commune ", <http://rue89.nouvelobs.com/2009/10/08/au-liban-durdenseigner-une-histoire-commune-dans-les-ecoles-120549>, consulté le 22 juin 2016.

Meijer, R. (2000), dir., Alienation or Integration of Arab Youth. Between Family, State and Street, Richmond, Curzon Press.

RAHEB, E. (2012), Sleepless nights, Beyrouth, 2h8min., <http://layalibalanoom. com>, consulté le 22 juin 2016.

Raphael, N. (2009), Trait d'union Islam-Christianisme, Beyrouth, Arab Printing Press.

SAGHiÉ, N. (2005), L’Orient-le-Jour, Beyrouth, 15 avril (archives électroniques). 
SKAFF, C. J. (2013), «L'amnistie et la justice transitionnelle. Un exemple: les accords de paix au Liban", Revue de philosophie et de sciences humaines, 31, p. 175-186, <https://leportique.revues.org/2686? lang=en>, consulté le 22 juin 2016.

Traoui, A. (2003), La mémoire de Beyrouth, Beyrouth, Banque de la Méditerranée.

\section{Résumé}

Bien que les pratiques de réconciliation connaissent une longue histoire au Liban, celles-ci se diversifient à partir des années 1990. Elles constituent un important objet d'étude pour de nombreux académiciens et académiciennes ainsi que chercheurs et chercheuses; elles sont devenues la cause commune d'une pléthore d'organismes non-gouvernementaux, d'associations civiles, de mouvements sociaux et d'artistes. Le thème de la réconciliation des Libanais et Libanaises sert aussi de cadre pour le discours politique. Cet article présente un état des lieux de ces pratiques en traitant premièrement de la relation de la réconciliation au dialogue interreligieux puis de la relation de la réconciliation à la mémoire nationale. Il présente par la suite certains exemples au sein de la société civile et identifie en conclusion quelques pistes de réflexion.

\section{Abstract}

Although reconciliation practices have a long history in Lebanon, they have been diversifying since the 1990s. Furthermore, they have become an important object of study for many scholars and researchers, and the common cause for numerous non-governmental organizations, civic associations, social movements and artists. The theme of reconciliation also serves as a framework for political discourse. This article presents first an overview of these practices by tackling the relationship between reconciliation, interreligious dialogue and national memory. It then highlights some examples found in the Lebanese civil society. It finally suggests some avenues to be explored. 